\title{
AVANÇOS E DESAFIOS NA ATENÇÃO À SAÚDE DE PESSOAS COM DEFICIÊNCIA NA ATENÇÃO PRIMÁRIA NO BRASIL: UMA REVISÃO INTEGRATIVA
}

\author{
E. G. AMORIM ${ }^{1}$, R. LIBERALI ${ }^{2}$, O. M. MEDEIROS NETA ${ }^{3}$ \\ ${ }^{1,3}$ Universidade Federal do Rio Grande do Norte, ${ }^{2}$ Universidade Federal de Santa Catarina \\ ericogur@gmail.com ${ }^{1}$
}

Submetido 03/11/2017 - Aceito 30/01/2018

DOI: $10.15628 /$ holos.2018.5775

\begin{abstract}
RESUMO
Trata-se de um estudo de revisão integrativa de literatura que objetivou descrever e analisar os fatores facilitadores e dificultadores para a atenção integral à saúde da pessoa com deficiência na atenção primária no Brasil, no período de 2006 a 2016. Pesquisou-se nas bases de dados Medical Literature Analysis and Retrieval System Online (MEDLINE), Literatura Latino-Americana e do Caribe em Ciências da Saúde (LILACS) e Scientific Electronic Library Online (SCIELO). Foram selecionados e submetidos à análise temática 30 estudos. As categorias que emergiram foram: 0 cuidado integral à saúde da pessoa com deficiência; Capacitação profissional para atuação junto à pessoa com deficiência e Acessibilidade aos serviços na atenção primária. A análise permitiu verificar que apesar de alguns avanços conquistados na
\end{abstract}

garantia da saúde à pessoa com deficiência, ainda são escassas as ações de seu cuidado e reabilitação na atenção primária. Além disso, as práticas profissionais ainda carecem de qualificação e capacitação para o cuidado integral pretendido e a falta de acessibilidade física e atitudinal nas unidades básicas de saúde perpetuam um ciclo de iniquidades historicamente construído. Conclui-se que para a inclusão das pessoas com deficiência se tornar uma realidade, é fundamental enfrentar o desafio ético, político e conjuntural da garantia da efetividade de seus direitos legalmente constituídos, articulando deste modo uma sociedade mais justa e humana na criação de novas formas de vida no encontro com o outro.

PALAVRAS-CHAVE: Atenção Primária à Saúde, Pessoa com Deficiência, Necessidade de Serviços de Saúde.

\section{ADVANCES AND CHALLENGES IN HEALTH CARE OF DISABLED PEOPLE IN BRAZILIAN PRIMARY HEALTH CARE SERVICES: AN INTEGRATIVE REVIEW}

\begin{abstract}
This is a study of integrative review of the literature which aimed to describe and analyze the factors that facilitate and impede the integral health care of the person with disability in the primary care in Brazil, from 2006 to 2016. It was researched on the data bases called Medical Literature Analysis and Retrieval System Online (MEDLINE), Latin American and Caribbean Literature in Health Sciences (LILACS) and Scientific Electronic Library Online (SCIELO). Thirty studies were selected and submitted to the thematic analysis. The categories which emerged were: The integral health care of the disabled person; Professional qualification to act with the disabled person and Accessibility to services in primary care. The analysis showed that despite some
\end{abstract}

advances achieved in guaranteeing the health of the disabled person, the actions of care and rehabilitation in primary care are still scarce. In addition, the professional practices still lack qualification and training for the intended integral care and the lack of physical and attitudinal accessibility in the basic health units perpetuate a cycle of inequities historically constructed. It is concluded that for the inclusion of people with disabilities to become a reality, it is fundamental to face the ethical, political and conjunctural challenge of guaranteeing the effectiveness of their legally constituted rights, thus articulating a more just and humane society in the creation of new forms of life in the encounter with the other.

KEYWORDS: Primary Health Care, People with Disabilities, Needs and Demands of Health Services. 


\section{APRESENTAÇÃO}

Mais de um bilhão de pessoas ou $15 \%$ da população mundial convivem com algum tipo de deficiência, conforme aponta World Health Organization (WHO), 2011. No Brasil, cerca de 45,6 milhões de pessoas (23,9\% da população) referiram ter alguma deficiência mental ou intelectual, motora, auditiva ou visual, de acordo com o censo do Instituto Brasileiro de Geografia e Estatística (IBGE) de 2010. Desse total, mais de 17,7 milhões (6,7\% da população) relataram alguma deficiência considerada severa (IBGE, 2010).

De acordo com a lei brasileira de inclusão, Lei № 13.146, de 6 de julho de 2015, no seu Artigo $2^{\circ}$, "considera-se pessoa com deficiência aquela que tem impedimento de longo prazo de natureza física, mental, intelectual ou sensorial, o qual, em interação com uma ou mais barreiras, pode obstruir sua participação plena e efetiva na sociedade em igualdade de condições com as demais pessoas". A referida lei explicita ainda que é dever do estado assegurar à pessoa com deficiência o direito à saúde por meio de uma "atenção integral em todos os níveis de complexidade, por intermédio do Sistema Único de Saúde (SUS), garantindo o acesso universal e igualitário" (Art. 18) e "promovendo o bem-estar pessoal, social e econômico" (Art. 8) (Brasil, 2015).

As principais causas de deficiência permanente são resultados de alterações genéticas, embriológicas, de doenças degenerativas, de acidentes de trânsito ou do envelhecimento humano (Diniz, Barbosa e Santos, 2009). Outros fatores relacionados ao número elevado de pessoas com deficiência no Brasil são a falta de saneamento básico, que ocasiona infecções; falta de assistência pré-natal e ao parto; os acidentes em ambientes de trabalho e a violência por arma de fogo (Figueira, 2014).

Pessoas com deficiência apresentam mais doenças crônicas e comorbidades, procuram mais internações e atendimentos ambulatoriais, além de haver maior índice de relatos de não recebimento de cuidados e de promoção à saúde do que por pessoas não deficientes (WHO, 2011). Adicionalmente, é aferido um coeficiente de mortalidade 22 vezes maior nas pessoas com deficiência que recebem o benefício assistencial (BPC) em relação à população brasileira (Cunha Falcão e França, 2016), justificando a necessidade de redução de tais desigualdades experimentadas no campo da saúde coletiva.

A Política Nacional de Saúde da Pessoa com Deficiência (Portaria MS/GM no 1.060, de 5 de junho de 2002) estabelece como propósitos gerais: "proteger a saúde da pessoa com deficiência; reabilitar a pessoa com deficiência na sua capacidade funcional e desempenho humano, contribuindo para a sua inclusão em todas as esferas da vida social; e prevenir agravos que determinem o aparecimento de deficiências". Entre as diretrizes da referida política, está a "melhoria paulatina do acesso às estruturas físicas, às informações e aos bens e serviços disponíveis aos usuários com deficiência no SUS, de modo a atender às necessidades particulares de todos os membros da sociedade" (Brasil, 2009).

No Brasil, a Atenção Básica desempenha papel central na garantia à população de acesso a uma atenção à saúde de qualidade, por ser o contato preferencial dos usuários, a principal porta de entrada e centro integrador de toda a Rede de Atenção à Saúde (Magalhães Junior, 2014). Para isso, orienta-se pelos princípios da universalidade, da acessibilidade, do vínculo, da 
continuidade do cuidado, da integralidade da atenção, da responsabilização, da humanização, da equidade e da participação social (Brasil, 2012).

As práticas de promoção e de assistência à saúde de pessoas com deficiências em serviços de atenção primária no país apontam para algumas indagações sobre a prestação do cuidado à saúde a esse público que vem se desenvolvendo nos últimos anos. Sendo assim, o objetivo desse estudo é descrever e analisar os fatores facilitadores e dificultadores mais recentes para a atenção integral à saúde da pessoa com deficiência no âmbito da atenção primária brasileira.

\section{METODOLOGIA}

Trata-se de uma pesquisa de revisão integrativa realizada no período de novembro e dezembro de 2016. Tal método é um tipo de revisão bibliográfica, que consiste na procura de referências teóricas para análise do problema de pesquisa e a partir das referências publicadas fazer as contribuições cientificas ao assunto em questão (Liberali, 2011).

A escolha do referido método de revisão integrativa deve-se ao fato de ser considerado o mais completo, pois permite a inclusão, síntese e incorporação na prática em saúde de pesquisas significativas com distintas metodologias e abordagens, assim como distintas formas de utilização da teoria, seja para promover ajustes aos dados coletados, seja como marco referencial (Souza, Silva \& Carvalho, 2010). Além disso, permite por meio da identificação de lacunas, um direcionamento para a realização de novas pesquisas (Mendes, Silveira e Galvão, 2008).

Para o levantamento dos artigos na literatura, realizou- se uma busca nas seguintes bases de dados eletrônicas: Medical Literature Analysis and Retrieval System Online (MEDLINE), Literatura Latino-Americana e do Caribe em Ciências da Saúde (LILACS) e Scientific Electronic Library Online (SCIELO), sendo complementada por busca manual nas referências descritas nos estudos selecionados. Foram utilizados, para busca dos artigos, os seguintes descritores e suas combinações nas línguas portuguesa e inglesa: "pessoas com deficiência" e "atenção primária à saúde", unidos pelo operador booleano "and".

Os critérios de inclusão definidos para a seleção dos artigos foram: artigos primários publicados em português, inglês ou castelhano, artigos na íntegra que retratam a temática referente à saúde da pessoa com deficiência na atenção primária brasileira e artigos publicados e indexados nos referidos bancos de dados nos últimos dez anos.

Para a extração das informações dos estudos selecionados foi utilizado um instrumento de coleta de dados, previamente elaborado abrangendo a base de dados, título do artigo, autores, periódico, ano, descritores, resumo, definição dos sujeitos, local do estudo, os objetivos, a metodologia empregada, tamanho da amostra, mensuração de variáveis, resultados e principais conclusões de cada estudo.

As análises dos dados foram sistematicamente processadas através do software NVIVO 10 for Windows, viabilizando a criação de dimensões e categorias, identificação de frequência das palavras mais recorrentes, análise de cluster das palavras e criação da árvore das palavras mais frequentes. 


\section{REVISÃO DA LITERATURA}

Foram identificados 94 artigos nas bases pesquisadas. Após a leitura dos títulos, resumos e palavras-chave e eliminando-se as duplicidades, totalizaram 30 artigos selecionados para análise, que foram lidos na íntegra.

Os periódicos que apresentaram o maior número de publicações selecionadas foram: Ciência e Saúde Coletiva e Revista de Terapia Ocupacional da Universidade de São Paulo, com cinco artigos cada. Quanto aos anos das publicações, os mais recorrentes foram: $2009(f=5), 2013$ $(f=5), 2014(f=4)$ e $2015(f=4)$. A Universidade de São Paulo foi a instituição com maior número de filiações dos autores (9), seguida pela Universidade Federal do Ceará, Universidade Estadual da Paraíba e Universidade Luterana do Brasil, com dois registros cada.

Os descritores mais comumente identificados foram: Atenção Primária à Saúde $(f=19)$, Pessoas com deficiência $(f=15)$, Acesso aos serviços de saúde ( $f=7)$, Enfermagem ( $f=5)$, Reabilitação $(f=5)$, Terapia ocupacional $(f=4)$, Saúde mental $(f=3)$ e Saúde da família $(f=3)$. Por seu turno, a análise dos resultados constantes nos resumos permitiu, por meio do software NVivo a identificação da nuvem de palavras (Figura 1) e das palavras mais recorrentes (Tabela 1).

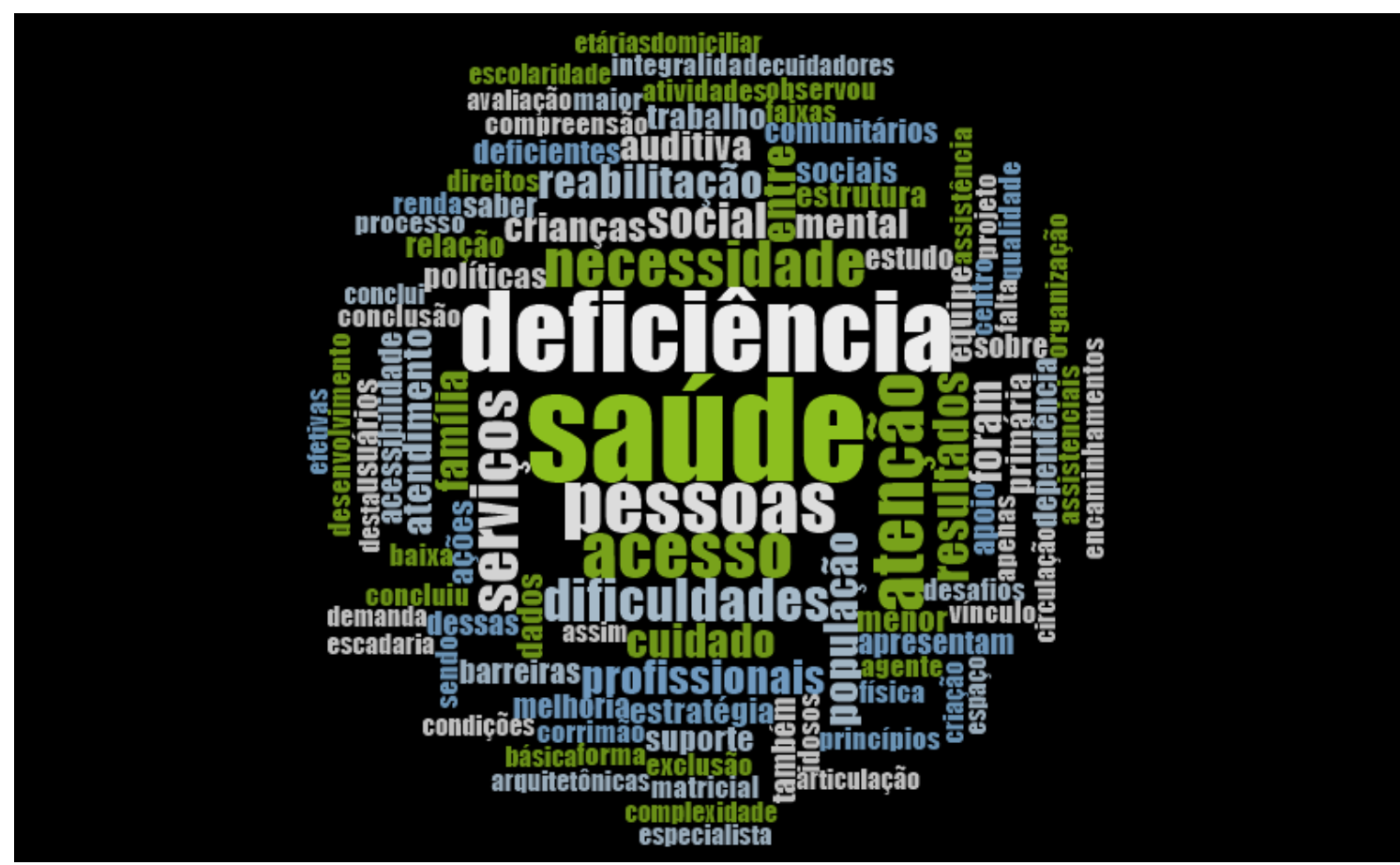

Figura 1: Nuvem de palavras extraída a partir do Software NVivo, utilizando os resultados dos artigos selecionados.

Fonte: Elaborado pelos autores (2017). 
Tabela 1: Frequência de palavras mais recorrentes obtida a partir do Software NVivo, utilizando os resultados dos artigos selecionados

\begin{tabular}{|c|c|c|c|c|}
\hline Palavra & Extensão & Contagem & $\begin{array}{c}\text { Percentual } \\
\text { ponderado (\%) }\end{array}$ & Palavras similares \\
\hline saúde & 5 & 44 & 1,69 & Saúde \\
\hline deficiência & 11 & 33 & 1,27 & deficência, deficiências \\
\hline pessoas & 7 & 23 & 0,88 & pessoa, pessoas \\
\hline acesso & 6 & 20 & 0,77 & Acesso \\
\hline atenção & 7 & 20 & 0,77 & Atenção \\
\hline serviços & 8 & 17 & 0,65 & serviço, serviços \\
\hline necessidade & 11 & 16 & 0,62 & necessidade, necessidades \\
\hline dificuldades & 12 & 14 & 0,54 & dificuldade, dificuldades \\
\hline resultados & 10 & 11 & 0,42 & resultados \\
\hline social & 6 & 11 & 0,42 & Social \\
\hline cuidado & 7 & 10 & 0,38 & cuidado, cuidados \\
\hline entre & 5 & 10 & 0,38 & Entre \\
\hline população & 9 & 10 & 0,38 & população \\
\hline profissionais & 13 & 10 & 0,38 & profissionais \\
\hline reabilitação & 12 & 10 & 0,38 & reabilitação \\
\hline família & 7 & 9 & 0,35 & família, famílias \\
\hline foram & 5 & 9 & 0,35 & Foram \\
\hline atendimento & 11 & 8 & 0,31 & atendimento \\
\hline auditiva & 8 & 8 & 0,31 & auditiva, auditivas \\
\hline crianças & 8 & 8 & 0,31 & criança, crianças \\
\hline mental & 6 & 8 & 0,31 & Mental \\
\hline ações & 5 & 6 & 0,23 & Ações \\
\hline dados & 5 & 6 & 0,23 & Dados \\
\hline equipe & 6 & 6 & 0,23 & equipe, equipes \\
\hline estratégia & 10 & 6 & 0,23 & estratégia, estratégias \\
\hline estrutura & 9 & 6 & 0,23 & estrutura, estruturas \\
\hline menor & 5 & 6 & 0,23 & menor \\
\hline políticas & 9 & 6 & 0,23 & política, políticas \\
\hline primária & 8 & 6 & 0,23 & primária \\
\hline sociais & 7 & 6 & 0,23 & sociais \\
\hline suporte & 7 & 6 & 0,23 & suporte \\
\hline trabalho & 8 & 6 & 0,23 & trabalho \\
\hline acessibilidade & 14 & 5 & 0,19 & acessibilidade \\
\hline apoio & 5 & 5 & 0,19 & Apoio \\
\hline apresentam & 10 & 5 & 0,19 & apresentam \\
\hline barreiras & 9 & 5 & 0,19 & barreiras \\
\hline comunitários & 12 & 5 & 0,19 & comunitário, comunitários \\
\hline
\end{tabular}

Com base nas informações obtidas a partir do processamento dos dados mencionado, realizamos o agrupamento nas seguintes categorias temáticas para estudo: O cuidado integral à saúde da pessoa com deficiência (17 artigos); Capacitação profissional para atuação junto à pessoa com deficiência (10 artigos); e Acessibilidade aos serviços na atenção primária 11 artigos), de modo que um mesmo artigo pode ser contemplado em mais de uma categoria. Em seguida, em cada uma das categorias temáticas que emergiram, foram extraídos os fatores facilitadores e dificultadores à 
efetivação da atenção à saúde das pessoas com deficiência no Brasil, a fim de responder ao objetivo desta pesquisa.

\subsection{0 cuidado integral à saúde da pessoa com deficiência}

Segundo Othero e Dalmaso (2009, p. 188), "a atenção primária à saúde da pessoa com deficiência deve incluir ações direcionadas a promoção da interação social, à construção de direitos e à transformação do lugar da deficiência". Para isso, o reconhecimento das necessidades de saúde como fenômeno afeto a formas particulares de produção e reprodução dos diferentes grupos sociais são prementes e podem ser identificadas a partir do "estabelecimento dos modos de viver, de trabalhar, de consumir, de se relacionar e das características do território" (Aoki e Correa, 2013).

De acordo com Silva (2011), a partir das necessidades dos indivíduos, das famílias e do meio social a prática do cuidado estabelece seus pilares de construção. Para esta efetivação, aspectos sociais, econômicos, familiares, biológicos, psicológicos e culturais devem interagir como mediadores nas particularidades de cada intervenção (Muniz Pinto et al., 2011).

A produção do cuidado integral no cotidiano assistencial deve ocorrer por meio das tecnologias leves que incluem o acolhimento, o vínculo, a corresponsabilização e autonomia, refletindo nas relações entre os meios da equipe e os usuários (Santos et al., 2007; Jorge et al., 2011) e são basilares para a humanização e reorientação do cuidado à saúde nas unidades básicas de saúde (Costa et al., 2009). Alinham-se à clínica ampliada, ao considerar o sujeito imerso em seu contexto e singularidade próprios, contrapondo-se ao modelo biomédico, centrado na doença e na fragmentação do cuidado (Campos, 2007).

A Estratégia de saúde da família (ESF) é um veículo prioritário de oferta e coordenação de cuidado aos usuários com deficiências, devendo promover a equidade e o acesso, além de garantir trajetórias terapêuticas balizadas por vulnerabilidades e potencialidades inerentes a cada sujeito, formulando projetos de intervenção individualizados (Figueredo e Costa, 2009). Também deverá estar atenta às opiniões, desejos e anseios particulares na elaboração do plano de ação para cada caso por meio de escuta qualificada e valorização da participação do usuário e de sua subjetividade (Jorge et al., 2015).

Uma importante estratégia compartilhada de cuidado de saúde aos usuários com deficiência entre as equipes de referência e equipes da atenção primária é o apoio matricial, viabilizado pelo princípio da intersetorialidade, visando aumentar a eficácia, eficiência e resolutividade pela equipe local por meio dos Núcleos de Apoio à Saúde da Família (NASF) (Silva, 2011; Campos e Domitti, 2007). Além de propiciar a corresponsabilização do cuidado e facilitar o contato direto entre os serviços, trata-se de um regulador de fluxos pactuado por profissionais e gestores com formas peculiares de configuração dependentes dos recursos disponíveis de cada loco região (Bezerra e Dimenstein, 2008).

Investigando os principais entraves e desafios para o funcionamento eficaz do apoio matricial, Hirdes (2015) demonstrou dificuldades no sistema de referência na rede de atenção à saúde; a ausência de diretrizes claras, construídas conjuntamente entre especialistas, generalistas e gestores; a sobrecarga de trabalho e ausência de reconhecimento e suporte por parte da gestão. Adicionalmente, Silva (2011) apontou o número insuficiente de profissionais apoiadores, a baixa coesão, comunicação e integração entre as distintas especialidades e 
profissões e a falta de capacitação permanente. Por fim, Jorge et al. (2015) reportaram a deficiência de espaço físico para realização das reuniões, a terceirização dos profissionais, a formação biomédica dos profissionais da atenção primária, e a desarticulação das políticas públicas, produzindo obstáculos à autonomia do sujeito e sua reabilitação.

As ações de reabilitação de pessoas com deficiência, como parte das intervenções em saúde, constituem uma das propostas a ser desenvolvidas pelos NASFs com vistas a promover ampliação da autonomia e independência, melhorar a qualidade de vida dos indivíduos e favorecer a sua participação social (Rocha e Kretzer, 2009). Além disso, tem o pressuposto de facilitar a integralidade, a equidade, a humanização dos serviços de saúde, combater a discriminação e ampliar o acesso irrestrito ao sistema de saúde (Brasil, 2008), realizando acompanhamento longitudinal das famílias, facilitando o acesso através de busca ativa e atendimento domiciliar (Souza e Rocha, 2010).

Entre as principais demandas e necessidades apresentadas por adultos com deficiência, estudar, realizar tratamento em reabilitação, ter uma moradia digna, alimento, trabalho, benefício previdenciário, deslocar-se, passear e conversar expressam não apenas acesso a direitos sociais, mas a própria manutenção na vida e sua realização enquanto projeto (Aoki e Correa, 2013). Nesse sentido, Schoeller et al. (2013), ao investigar aspectos da funcionalidade das pessoas com deficiência, por meio da medida de independência funcional, conferiram dependência na totalidade do grupo avaliado, especialmente para locomoção, comprovando importância de ações de reabilitação e da aplicação da referida medida nos serviços de atenção primária a saúde no planejamento do cuidado às pessoas dependentes.

Van Schaik et al. (2014), avaliando um grupo de crianças com deficiência que utilizaram a atividade lúdica promovida pela terapia ocupacional como estratégia de reabilitação, notaram melhora do desenvolvimento neuropsicomotor, além da criação de redes de suporte entre os pais. Isso reforça a ideia de abordar o atendimento à infância de maneira ampliada e contribui na constituição de possibilidades de inclusão social e de organização de redes de apoio.

Considerando ainda o fato de que pessoas com deficiências podem sofrer impedimentos que dificultam sua autonomia e vivência em sociedade e a precariedade ainda presente no acesso aos serviços de reabilitação no SUS, uma ferramenta de intervenção utilizada pela equipe de reabilitação como estratégia para facilitar oportunidades de convivência de pessoas com deficiências, por meio da ampliação e ativação das relações sociais significativas é a atenção domiciliar, permitindo diminuição do impacto da deficiência na vida diária (Ferreira e Oliver, 2010). A esse exemplo, Souza e Rocha (2010), ao analisar as ações da equipe de reabilitação, identificaram o enfrentamento da baixa qualidade de vida da população usuária com deficiência por meio de realização de acompanhamento longitudinal das famílias e facilitação do acesso através de busca ativa e atendimento domiciliar, levando em conta as especificidades encontradas como referências para a organização do cuidado.

\subsection{Capacitação profissional para atuação junto à pessoa com deficiência}

Apesar de a deficiência e reabilitação serem rotineiramente atribuídas por profissionais de saúde e pela população como de responsabilidade dos níveis de maior complexidade ou de instituições filantrópicas, a Estratégia Saúde da Família pretende ser um espaço apropriado para atenção às necessidades de saúde das pessoas com deficiência (Souza e Rocha, 2010; Antunes e Rocha, 2011) Para que isso se concretize, a prática profissional dos que atuam junto à pessoa 
com deficiência deve constituir-se em um trabalho coletivo e de cooperação mútua entre sujeitos, compondo uma teia de relações alimentada por interação e diálogo, batizado em uma escuta ativa e qualificada, consistindo em dilemas éticos, técnicos e políticos a serem enfrentados (Martini, 2008).

Algumas experiências de capacitação são observadas no âmbito da atenção primária, de modo insipiente, mas protótipos para novas experimentações, avanços e discussões no tocante à atenção à saúde da pessoa com deficiência. Nessa direção, Maia et al. (2014), ao avaliarem as facilidades e fragilidades concernentes à aquisição de competências e habilidades por agentes comunitários de saúde em atividade após curso de capacitação para atuar junto às pessoas com deficiência, demonstraram significativa apreensão pelos agentes dos conceitos de deficiência física, auditiva, visual e deficiência múltipla. Porém, houve dificuldade na assimilação de conceitos mais abstratos como deficiência, necessidades especiais, desvantagem, pessoa cega e baixa visão, refletindo em um pior preenchimento das fichas de cadastros pelos agentes junto aos deficientes visuais com índice de erro de 15,3\%.

Em outro estudo, França et al. (2012), ao enumerar facilidades e dificuldades para atender às demandas de usuários com lesão medular em uma unidade de saúde da família, detectaram "desqualificação profissional para a assistência e um cuidado fragmentado e biologista, compondo desafios à construção de práticas de saúde efetivas e resolutivas". Tedesco e Junges (2013), por sua vez, ao apontar as dificuldades que os profissionais da saúde comunitária vivenciam no atendimento aos usuários surdos, evidenciaram "sentimentos de desconforto, ansiedade e angústia dos profissionais como fatores presentes nos atendimentos, porque se sentem despreparados"; Também referiram à "necessidade de uma postura de disponibilidade e dedicação, incluindo a busca de aperfeiçoamento por meio de cursos e capacitações".

Por meio de espaços de capacitação e estratégias para reflexão diante dos problemas e desafios da saúde pública nos municípios e regiões, é possível enfrentar o desafio de produzir transformações nas práticas profissionais e na própria organização do trabalho (Batista e Gonçalves, 2011). Nesse diapasão, a educação permanente em saúde é uma condição para o desenvolvimento de uma escuta qualificada e de uma produção das aprendizagens de cuidado, relativas à intervenção no andar da vida individual e coletiva (Ceccim, 2005), deverá, portanto, ser orientada pela maior resolutividade dos problemas de saúde das populações, ocupando um lugar central e finalístico, nas políticas de saúde, permitindo a construção da acessibilidade da atenção e do sistema de saúde, incluindo às pessoas com deficiência, entendidos como cidadãos de direitos (Ceccim, 2004/2005).

\subsection{Acessibilidade aos serviços na atenção primária}

Apesar da fundamental ampliação de cobertura da atenção básica nacionalmente observada nos últimos anos, como uma das dimensões do acesso aos serviços de saúde ainda são muitos os fatores que dificultam a entrada e a permanência das pessoas com deficiência no sistema de saúde, o que limitam o direito da assistência universal constitucionalmente garantido (Albuquerque et al., 2014; Martins et al., 2016). Além disso, as pessoas com deficiência, ao apresentarem características particulares e diferenciadas da população em geral, enfrentam mais barreiras a serem transpostas na sociedade, problema este considerado central para a consolidação do Sistema Único de Saúde (França e Pagliuca, 2009). 
Segundo Othero e Dalmaso (2009), o acesso configura-se como uma das principais necessidades das pessoas com deficiência. Entretanto, essa população caracterizada por um complexo multifacetado de exclusão e privação coletiva experimenta dificuldades em relação ao acesso aos serviços de saúde, o que denuncia a iniquidade, a desigualdade e a falta de acessibilidade, vertentes potencializadoras do isolamento (Souza e Pimentel, 2012).

Avaliando-se as dificuldades no acesso de pessoas com deficiência aos serviços de saúde da atenção primária, a partir de dados do Programa Nacional de Melhoria do Acesso e da Qualidade da Atenção Básica (PMAQ-AB), Albuquerque et al. (2014) observaram diversas barreiras arquitetônicas nas unidades básicas de saúde como a inexistência de banheiros adaptados, a ausência de barras de apoio e corrimões, além de baixa capacidade de acomodação de cadeiras de rodas na sala de espera. Em outro estudo, Siqueira et al. (2009), ao descrever as condições arquitetônicas de 240 unidades básicas de saúde em sete estados do país, demonstraram inadequação em cerca de $60 \%$ das UBS, devido a presença de degraus, falta de corrimãos, rampas, banheiros adaptados para os cadeirantes e salas de espera inadequadas às necessidades de saúde das pessoas com deficiência.

Em conformidade a essa temática, Martins et al. (2016), ao aplicar checklist fundamentado na Norma Técnica 9050 da ABNT em 90 unidades básicas de saúde, aferiram que o acesso se dá por escadaria sem corrimão em 28,9\%; escadaria com corrimão em 6,7\% e através de rampa de acesso apenas em 47,8\% das USF. Utilizando-se de metodologia afim, França et al. (2012), em 20 unidades básicas de saúde, ressaltaram a violência simbólica a qual a ambiência e o acesso às pessoas com deficiência estão permeados, a partir da presença de barreiras arquitetônicas e atitudinais às pessoas com deficiência. Essa realidade expressa a não valorização da demanda, contribuindo para a complicação no estado de saúde, tendo o descaso e o desconhecimento de sua legislação de amparo forte aliados.

Ao buscar compreender como ocorre a acessibilidade das crianças com deficiência à atenção primária em saúde, por meio de entrevista com profissionais do serviço, Rosário et al. (2013) apresentaram barreiras estruturais, geográficas, organizacionais e atitudinais, incluindo a falta de infraestrutura adequada nas unidades de saúde, dificuldades na marcação das consultas para prioridade de atendimento e entraves no deslocamento nas vias públicas para a unidade, afetando negativamente a qualidade da assistência à saúde ofertada aos usuários, com prejuízos ao desenvolvimento de suas potencialidades. Tal fato foi corroborado por Souza e Rocha (2010), ao levar em conta, diferentes discursos, posições sociais e origens culturais, os aspectos da acessibilidade arquitetônica e urbana, foram denunciados como fatores importantes na determinação da possibilidade de acessar os serviços de saúde, por pessoas com deficiência, com reflexos diretos na qualidade de vida, uma vez que há pouca responsabilização e engajamento social e estatal na luta pela eliminação das barreiras físicas e atitudinais nos espaços públicos.

Deste modo, as dificuldades de acesso a serviços de atenção primária à saúde e de reabilitação são fatores de expressão ligados a determinantes sociais da saúde, contribuindo para índices de iniquidades sociais, havendo necessidade de elaboração de programas para a inclusão social e acessibilidade em articulação à estratégia saúde da família (Fiorati e Elui, 2015). Para que as pessoas com deficiência recebam assistência equitativa, igualitária e universal, conforme preconizado pelo SUS, a partir de ações facilitadoras de inclusão e acessibilidade, seus direitos precisam ser efetivamente implementados e fiscalizados, expandindo, com isso, as capacidades 
de fazer frente às limitações presentes pela condição física e/ou psicológica e garantindo saúde, dignidade às pessoas e, acima de tudo, justiça social (Lopes e Bohusch, 2014).

\section{CONSIDERAÇÕES FINAIS}

Entre os fatores facilitadores para o alcance das estratégias de cuidado observadas à saúde da pessoa com deficiência, as ações de reabilitação, a visita domiciliar e o apoio matricial são basilares da integralidade da atenção. Apesar disso, como fatores dificultadores deste cuidado, ainda é notável a baixa qualificação dos profissionais da atenção primária à saúde para as demandas dos usuários com alguma deficiência, o que requer espaços regulares de capacitação.

Por sua vez, a falta de acessibilidade nos serviços de saúde também se demonstrou como fator a dificultar o alcance da integralidade em saúde, salvaguardada pelo arcabouço jurídiconormativo brasileiro. Sob esse plano de fundo, ainda são presentes barreiras físicas e atitudinais nas maiorias das unidades básicas de saúde, contribuindo para uma ambiência permeada por violência simbólica às pessoas com alguma limitação ou impedimento físico, mental ou sensorial. Esse fato perpetua um ciclo de iniquidades no qual as pessoas com deficiência estão imersas, contribuindo para a exclusão social.

Ao considerar os desafios postos à efetivação do direito à saúde das pessoas com deficiência, sob o ângulo coletivo, consubstanciado nos princípios de dignidade da pessoa humana e equidade social, salientamos a premente necessidade de colocarmos em prática o que preceitua o corpus de lei sob o qual as pessoas com deficiência são amparadas, a partir de sólidas e resolutivas políticas públicas voltadas à melhoria da qualidade de vida desta população, reparando uma desigualdade de oportunidades por muito tempo negligenciada pelo estado democrático de direito.

\section{REFERÊNCIAS}

Albuquerque, M. D. S. V. D. et al. (2014). Acessibilidade aos serviços de saúde: uma análise a partir da Atenção Básica em Pernambuco. Saúde debate, 38(spe), 182-194.

Antunes, M. H., \& Rocha, E. F. (2011). Desbravando novos territórios: incorporação da Terapia Ocupacional na estratégia da saúde da família no município de São Paulo e a sua atuação na atenção à saúde da pessoa com deficiência no período de 2000-2006. Revista de Terapia Ocupacional da Universidade de São Paulo, 22(3), 270-278.

Aoki, M., \& Correa, F. O. (2013). Pessoas com deficiência moradoras de bairro periférico da cidade de São Paulo: estudo de suas necessidades. Cadernos de Terapia Ocupacional da UFSCar, 21(2), 391-398.

Batista, K. B. C., \& Gonçalves, O. S. J. (2011). Formação dos profissionais de saúde para o SUS: significado e cuidado. Saúde e Sociedade, 20(4), 884-899.

Bezerra, E., \& Dimenstein, M. (2008). O CAPS e o Trabalho em Rede: tecendo o Apoio Matricial na Atenção Básica. Psicologia, Ciência e Profissão, 28(3), 632-645. 
Brasil. Ministério da Saúde. (2008). Portaria 154 de 24 de janeiro de 2008 cria os NASFs - Núcleo de Atenção Integral à Saúde da Família. Recuperado em 17 dezembro, 2016, de http://bvsms.saude.gov.br/bvs/saudelegis/gm/2008/prt0154_24_01_2008.html.

Brasil. Ministério da Saúde. (2009). Atenção à Saúde da Pessoa com Deficiência no Sistema Único de Saúde - SUS. Brasília: Ministério da Saúde. Recuperado em 17 novembro, 2016, de http://portal.saude.gov.br/portal/arquivos/pdf/atensaudecomdefic.pdf.

Brasil. Ministério da Saúde. Secretaria de Atenção à Saúde. Departamento de Atenção Básica. (2012). Política Nacional de Atenção Básica. Ministério da Saúde. Secretaria de Atenção à Saúde. Departamento de Atenção Básica. (p. 110). (Série E. Legislação em Saúde). Brasília: Ministério da Saúde. Recuperado em 17 novembro, 2016, de http://189.28.128.100/dab/docs/publicacoes/geral/pnab.pdf.

Brasil. (2015). Lei no 13.146, de 6 de julho de 2015. Institui a Lei Brasileira de Inclusão da Pessoa com Deficiência (Estatuto da Pessoa com Deficiência). Recuperado em 20 novembro, 2016, de http://www.planalto.gov.br/ccivil_03/_Ato2015-2018/2015/Lei/L13146.htm.

Campos, G. W. S. (2007). Reflexões sobre a clínica ampliada em equipes de saúde da família. In: Campos GWS. Saúde Paidéia. (3a ed.). (p. 68-75). São Paulo: Hucitec.

Campos, G. W. S., \& Domitti, A. C. (2007). Apoio matricial e equipe de referência: uma metodologia para gestão do trabalho interdisciplinar em saúde. Cadernos de Saúde Pública, 23 (2), 399-407.

Ceccim, R. B. (2004/2005) Educação Permanente em Saúde: desafio ambicioso e necessário. Interface comun saúde educ, 9(16), 161-177.

Ceccim, R. B. (2005). Réplica. Interface comun saúde educ, 9(16), 175-177. Recuperado em 20 dezembro, 2016, de http://www.scielosp.org/scielo.php?script=sci_arttext\&pid=S1414$32832005000100016 \& \operatorname{lng}=e n \& n r m=$ iso.

Costa, G. D. et al. (2009). Saúde da família: desafios no processo de reorientação do modelo assistencial. Rev Bras Enferm, 62(1),113-8.

Cunha Falcão, S., \& de França, I. S. X. (2016). Morbidade e mortalidade em pessoas com deficiência na Previdência Social do Brasil. Ciência, Cuidado e Saúde, 15(2), 242-249.

Diniz, D., Barbosa, L., \& Santos, W. R. (2009). Deficiência, direitos humanos e justiça. Sur. Rev. Int. direitos humanos, São Paulo, 6 (11) 64-77. Recuperado em 16 novembro, 2016, de http://www.scielo.br/scielo.php?script=sci_arttext\&pid=S1806$64452009000200004 \& \operatorname{lng}=e n \& n r m=$ iso.

Ferreira, T. G., \& Oliver, F. C. (2010). A atenção domiciliar como estratégia para ampliação das relações de convivência de pessoas com deficiências físicas. Revista de Terapia Ocupacional da Universidade de São Paulo, 21(3), 189-197.

Fiorati, R. C., \& Elui, V. M. C. (2015). Social determinants of health, inequality and social inclusion among people with disabilities. Revista latino-americana de enfermagem, 23(2), 329-336.

França, I. S. X., \& Pagliuca, L. M. F. (2009). Inclusão social da pessoa com deficiência: conquistas, desafios e implicações para a enfermagem. Rev Esc Enferm da USP, 43(1),178-85.

França, I. S. X. et al. (2012). O descuidar do lesado medular na atenção básica: desafios bioéticos para as políticas de saúde. Rev. bras. enferm, 65(2), 236-243. 
Figueira, E. (2014). Introdução à psicologia e pessoas com deficiência: a construção de um novo relacionamento! 2a. ed. São Paulo: Edição do Autor/AgBook.

Figueredo, M. D., \& Campos, R. O. (2009). Saúde mental na atenção básica à saúde de Campinas, SP: uma rede ou um emaranhado? Ciênc Saúde Coletiva. 14(1),129-38.

Instituto Brasileiro de Geografia e Estatística [IBGE] (2010). Censo Demográfico 2010. Recuperado em 17 novembro, 2016, de http://censo2010.ibge.gov.br.

Jorge, M. S. B. et al. (2011) Promoção da saúde mental - tecnologias do cuidado: vínculo, acolhimento, co-responsabilização e autonomia. Cien Saude Colet, 16(7),3051-60.

Jorge, M. S. B. et al. (2015). Apoio matricial, projeto terapêutico singular e produção do cuidado em saúde mental. Texto \& Contexto Enfermagem, 24(1), 112-120.

Hirdes, A. (2015). A perspectiva dos profissionais da Atenção Primária à Saúde sobre o apoio matricial em saúde mental. Cien Saude Colet, 20(2), 371-382.

Liberali, R. (2011). Metodologia Científica Prática: um saber-fazer competente da saúde à educação. 2a ed. rev. ampl. (p. 206). Florianópolis: Postmix.

Lopes, M. J. M. \& Bohusch, G. (2014). Usuários portadores de deficiência: questões para a atenção primária de saúde. Revista Baiana de Enfermagem, 28(1).

Magalhães Junior, H. M. (2014). Atenção básica enquanto ordenadora da rede e coordenadora do cuidado: ainda uma utopia?. Divulg Saúde debate, 51, 14-29.

Maia, E. R., Pagliuca, L. M. F., \& Almeida, P. C. D. (2014). Learning of community health agent to identify and register disabled people. Acta Paulista de Enfermagem, 27(4), 326-332.

Martini, J. G. (2008). Mas, do que é mesmo que estamos falando quando abordamos a integralidade? Rev Bras Enferm, 61(3), 285.

Martins, K. P. et al. (2016). Internal structure of Family Health Units: access for people with disabilities. Ciência \& Saúde Coletiva, 21(10), 3153-3160.

Mendes, K. D. S., Silveira, R. C. D. C. P., \& Galvão, C. M. (2008). Revisão integrativa: método de pesquisa para a incorporação de evidências na saúde e na enfermagem. Texto \& ContextoEnfermagem, 17(4), 758-764.

Muniz, D. P. et al. (2011). Projeto terapêutico singular na produção do cuidado integral: uma construção coletiva. Texto Contexto Enferm, 20(3),293-302.

Othero, M. B., \& Dalmaso, A. S. W. (2009). Pessoas com deficiência na atenção primária. Interface. Comunicação Saúde Educação, Botucatu, 13(28), 177-88.

Rocha, E. F., \& Kretzer, M. R. (2009). Ações de reabilitação de pessoas com deficiência na estratégia da saúde da família da Fundação Zerbini e Secretaria Municipal de Saúde de São Paulo-Região Sudeste-Sapopemba/Vila Prudente-período 2000/2006. Revista de Terapia Ocupacional da Universidade de São Paulo, 20(1), 59-67.

Rosário, S. S. D. D. et al. (2013). Acessibilidade de crianças com deficiência aos serviços de saúde na atenção primária. Rev. eletrônica enferm, 15(3), 740-746.

Santos, A. M. et al. (2007). Linhas de tensões no processo de acolhimento das equipes de saúde bucal do Programa Saúde da Família: o caso de Alagoinhas, Bahia, Brasil. Cad Saúde Pública, 23(1),75-85. 
Schoeller, S. D. et al. (2013). Aspectos da (in) dependência funcional de pessoas adscritas a um centro de saúde. Ciência, Cuidado e Saúde, 12(1), 47-55. Recuperado de DOI: 10.4025/cienccuidsaude. v12i1. 18026.

Silva, D. S. D. (2011). Apoio matricial em saúde mental: uma análise sob ótica dos profissionais de saúde da atenção primária. Revista Portuguesa de Enfermagem de Saúde Mental, (6), 20-27.

Siqueira, F. C. V. et al. (2009). Barreiras arquitetônicas a idosos e portadores de deficiência física: um estudo epidemiológico da estrutura física das unidades básicas de saúde em sete estados do Brasil. Cien Saude Colet, 14(1), 39-44.

Souza, F. D. R., \& Pimentel, A. M. (2012). Pessoas com deficiência: entre necessidades e atenção à saúde. Cad. Ter. Ocup. UFSCar, 20(2).

Souza, M. T., da Silva, M. D., de Carvalho, R. (2010). Revisão integrativa: o que é e como fazer. Einstein, 8 (1) , 102-106.

Souza, C. C. B. X., \& Rocha, E. F. (2010). Portas de entrada ou portas fechadas?: o acesso à reabilitação nas Unidades Básicas de Saúde da região sudeste do município de São Pauloperíodo de 2000 a 2006. Revista de Terapia Ocupacional da Universidade de São Paulo, 21(3), 230-239.

Tedesco, J. D. R., \& Junges, J. R. (2013). Desafios da prática do acolhimento de surdos na atenção primária. Cad. Saúde Pública, Rio de Janeiro, 29(8),1685-1689.

Van Schaik, E. E. et al. (2014) Reflexões sobre a atenção às crianças com deficiência. Rev Ter Ocup Univ São Paulo. 25(3), 233-241.

World Health Organization [WHO]. (2011). World report on disability 2011. (pp. 323-325). WHO: Malta. Recuperado em 17 novembro, 2016, de http://www.who.int/disabilities/world_report/2011/report.pdf. 